\title{
Species of the subfamily Triatominae Jeannel, 1919 (Hemiptera: Reduviidae) present in the Collection of Chagas Disease Vectors (FIOCRUZ-COLVEC), State of Minas Gerais
}

\author{
Rita de Cássia Moreira de Souza ${ }^{[1]}$, Raissa Nogueira Brito ${ }^{[1]}$, \\ Anatiele Borges Barbosa ${ }^{[1]}$ and Liléia Diotaiuti ${ }^{[1]}$
}

[1]. Laboratório de Triatomíneos e Epidemiologia da Doença de Chagas, Centro de Pesquisas René Rachou, Fundação Oswaldo Cruz, Belo Horizonte, MG.

\begin{abstract}
Introduction: Biological collections are depositories of information on different species and contribute to the knowledge, protection, conservation and maintenance of biodiversity. Methods: A list of triatomine species currently included in the Collection of Chagas Disease Vectors (FIOCRUZ-COLVEC) was prepared from the database made available by the Reference Center on Environmental Information. Results: COLVEC curatorship houses 4,778 specimens of triatomines, of which 811 come from other American countries (Argentina, Bolivia, Colombia, Costa Rica, the United States of America, Guatemala, Mexico, Peru, Uruguay and Venezuela) and 3,967 are autochthonous from Brazil. Altogether, 56 species of Chagas disease vectors are represented in the COLVEC: two species of the Tribe Cavernicolini Usinger, 1944; fifteen species of the tribe Rhodniini Pinto, 1926 , of which 12 are of the genus Rhodnius and 3 are of the genus Psamolestes; and 39 species of the tribe Triatomini Jeannel, 1919, represented by the genus Dipetalogaster, two species of the genus Eratyrus, two of the genus Meccus, seven of the genus Panstrongylus and 27 of the genus Triatoma. Conclusions: This list provides important data on the diversity of triatomines currently included in COLVEC, including the expanded area of Panstrongylus lutzi occurrence in the municipalities Pirapora and Januária, State of Minas Gerais. The maintenance and expansion of the collection ensures the preservation of biodiversity and further studies.
\end{abstract}

Keywords: Zoological collection. Triatominae. Biodiversity. Medical entomology. Systematics. Taxonomy.

\section{INTRODUCTION}

Biological collections are depositories of information on different species and contribute to the knowledge, protection, conservation and maintenance of biodiversity ${ }^{1}$. Due to the importance of biological collections for a nation's scientific knowledge and development, as well as the global concern for the preservation of biodiversity, collections are currently considered an inalienable heritage of the state and of the hosting institutions. Collections are assigned this importance because they house taxonomic data on the species and information related to the environment, enabling, albeit indirectly, the acquisition of knowledge of different regions of the planet.

The Oswaldo Cruz Foundation has created the Permanent Forum of Biological Collections, which assessed and conclusively interpreted the collections of each regional unit and

Address to: Dra Rita de Cássia Moreira de Souza. Laboratório de Triatomíneos e Epidemiologia de Doença de Chagas/CPqRR/FIOCRUZ. Avenida Augusto de Lima 1715, 30190-002 Belo Horizonte, MG, Brasil.

Phone: 5531 3349-7801

e-mail: rita@cpqrr.fiocruz.br

Received 12 September 2014

Accepted 24 November 2014 granted institutional recognition for those meeting the criteria of the analysis ${ }^{2}$. The Collection of Chagas Disease Vectors (Fiocruz-COLVEC), located in the Laboratory of Triatomines and Epidemiology of Chagas Disease [Laboratório de Triatomíneos e Epidemiologia da Doença de Chagas (LATEC)] of the René Rachou Research Center [Centro de Pesquisas René Rachou (CPqRR/FIOCRUZ)] underwent the process of institutional recognition and approval. Recently recognized by the Ministry of Environment as a Depository of Genetic Heritage Component Samples (Federal Official Gazette No. 168, August 29, 2012, Section 3, page 123), the collection houses different populations of triatomines from Argentina, Brazil, Bolivia, Colombia, Costa Rica, the United States of America, Guatemala, Mexico, Peru, Uruguay and Venezuela.

Collection of Chagas Disease Vectors was started through a donation from the private collection of Dr. Hélio Noguera Espínola in 1996. Comprising 286 species, this collection is important not only for its historical and affective value but also because it hosts triatomine populations from different geographic regions, including areas that are currently completely urbanized. Most of the collection was gathered after the 1990s, and it mainly consists of voucher specimens from research projects carried out by LATEC. In addition, the collection holds an increasing number of species obtained in close collaboration with the Triatomine and Epidemiology of Chagas Disease Reference Laboratory (Laboratório de 
Referência de Triatomíneos e Epidemiologia da Doença de Chagas - FIOCRUZ/Ministry of Health, Secretary of Health Surveillance). In this regard, the importance of the work carried out by COLVEC in collaboration with the Reference Service and the Regional Departments of Health from municipalities in the State of Minas Gerais is noteworthy for promoting consolidation within the perspectives of the Brazilian Unified Health System (SUS).

The demand for data associated with these species, including from a growing non-taxonomist audience, has been increasing ${ }^{1}$. Moreover, information must be readily available both to save time in the search for information on the tags attached to specimens and to maintain the integrity of the specimens by avoiding excessive handling. Thus, the computerization of biological collections has been a priority in many institutions to address access and organization and to protect the collections ${ }^{3}$.

Data input on the specimens in a digitalized database is the most efficient method of making this information available to a wide audience and ensuring visibility and access and thereby facilitating the dissemination of data. The present work aims to report the number of species currently included in the COLVEC collection.

\section{METHODS}

The collection is archived in good storage conditions in LATEC in a dedicated room. Insects are preserved dry; adults (males and females) and fourth and fifth stage nymphs are mounted on entomological pins, whereas eggs and nymphs from first to third stage are glued onto cardboard triangles and pinned.

In addition to the conventional registry in the record book, COLVEC's specimens are cataloged in a database managed by the Reference Center on Environmental Information (CRIA), thus ensuring their access and visibility. Both the record book and the database contain specific information on each specimen, such as data on the collector, collection location, date of collection, and name of the investigator who provided the identification and taxonomic data. These data are provided by the curator of the collection. The database is available for consultation online on CRIA's website, integrated with the speciesLink network (http://splink.cria.org.br), and COLVEC's website (http://colvec.fiocruz.br/index). The lists of triatomines presented below were prepared based on the first database.

\section{RESULTS}

Among the 4,778 specimens registered in the CRIA database, 811 originated from other countries (Argentina, Bolivia, Colombia, Costa Rica, the United States of America, Guatemala, Mexico, Peru, Uruguay and Venezuela) as Table 1 and Table 2. Thus, most of the collection consists of triatomine species native to Brazil, with 18 of its 27 states represented in the collection (Table 3 and Table 4). The States of Ceará and Minas Gerais are particularly well represented, contributing 952 and 2,071 specimens, respectively (Figure 1). The large number of specimens from these states is due to the partnership between LATEC and the two state health departments, which is essential for conducting projects. With regard to populations, the number of specimens from Minas Gerais confers a peculiar property to the collection: the regionalized character of the triatomine fauna.

In short, the collection is composed of two species of the tribe Cavernicolini Usinger, 1944; fifteen species of the tribe Rhodniini Pinto, 1926, of which 12 are of the genus Rhodnius and three are of the genus Psammolestes; and 39 species are of the tribe Triatomini Jeannel, 1919, represented by the genus Dipetalogaster, two species of the genus Eratyrus, two species of the genus Meccus, seven of the genus Panstrongylus and 27 of the genus Triatoma.

In the following list, only native species from Brazil are presented. These species are organized in alphabetical order by tribe, genus, species and sex. Whenever possible, the state and locality of capture in Brazil were also added, followed by the total number of specimens.

\section{DISCUSSION}

Considering that the conservation of biodiversity is a common concern of humankind, biological collections must serve as guardians of ex situ biological heritage. In the broadest sense of their use, such collections serve as basis for scientific research and educational activities and can provide valuable material of historical importance 4 . Thus, FIOCRUZ holds diverse collections that are important information sources for the reconstruction of the history of biological and biomedical sciences in Brazil ${ }^{5}$.

The primary mission of COLVEC's curatorship is based on the principles of the organization and permanent preservation of biological material ${ }^{6,7}$, accessibility to material and associated information $^{8}$ and ensuring the taxonomic identification of the Triatominae. However, other related activities are worth noting: 1) supporting research, as the collection is responsible for the legal deposit of voucher specimens from projects; 2) supporting the Reference Service through training courses and the identification of Triatominae; 3) making the collection available for public research through universities, research centers and museums; 4) contributing to the training of human resources through the guidance of students; and 5) participating in scientific and educational events promoting conservation awareness.

With a total capacity for 15,000 specimens, there are currently 5,467 entries in COLVEC's record book. This number will likely continuously increase due to research activities, the Reference Service and the collection's recent accreditation as a Depository of Genetic Heritage Component Samples. The exponential growth of the collection is expected to ensure a greater depiction of the diversity of the Brazilian triatomine fauna as well as of other countries where Chagas disease is endemic. The largest number of specimens and the different origins of a species can enable studies on intraspecific variability, with morphological variations observed dynamically throughout the species' distribution area. 
TABLE 1 - Contribution of triatomine fauna from other American countries to the COLVEC collection (Tribe Cavernicolini Usinger, 1944 and Tribe Rhodniini Pinto, 1926). Information in parentheses includes the specimen number and sex.

\begin{tabular}{|c|c|c|c|c|}
\hline Tribe & Species & Country & $\begin{array}{c}\text { Province, } \\
\text { Department or } \\
\text { State }\end{array}$ & County (sex) \\
\hline $\begin{array}{l}\text { Cavernicolini } \\
\text { Usinger, } 1944\end{array}$ & Cavernicola pilosa Barber, 1937 & Venezuela & Portuguesa & Tierra Buena $(5 \mathrm{M})$ \\
\hline \multirow{28}{*}{ Rhodniini Pinto, 1926} & $\begin{array}{c}\text { Rhodnius colombiensis } \\
\text { Mejia, Galvão \& Jurberg, } 1999\end{array}$ & Colombia & Tolima & Coyaima $(2 \mathrm{~F})$ \\
\hline & $\begin{array}{l}\text { Rhodnius ecuadoriensis } \\
\text { Lent \& León, } 1958\end{array}$ & Peru & NI & $\mathrm{NI}(2 \mathrm{~F}, 3 \mathrm{M})$ \\
\hline & \multirow{2}{*}{ Rhodnius neivai Lent, 1953} & \multirow{2}{*}{ Venezuela } & Lara & $\mathrm{NI}(6 \mathrm{~F}, 1 \mathrm{M}, 6 \mathrm{SE})$ \\
\hline & & & NI & NI (1M) \\
\hline & Rhodnius pallescens Barber 1932 & Colombia & NI & NI $(2 M)$ \\
\hline & \multirow{3}{*}{ Rhodnius pictipes Stål, 1872} & \multirow{3}{*}{ Venezuela } & Cojedes & $\begin{array}{l}\text { Manrrique }(2 \mathrm{~F}, 2 \mathrm{M}) \text {; San Carlos } \\
(2 \mathrm{~F}, 2 \mathrm{M}) \text {; Tierra Caliente }(3 \mathrm{~F}, 2 \mathrm{M}) \text {; } \\
\text { Tinaquillo (1M) }\end{array}$ \\
\hline & & & Portuguesa & $\mathrm{NI}(1 \mathrm{M})$ \\
\hline & & & $\mathrm{NI}$ & $\mathrm{NI}(1 \mathrm{~F}, 2 \mathrm{M})$ \\
\hline & \multirow{13}{*}{ Rhodnius prolixus Stål, 1859} & \multirow{5}{*}{ Peru } & $\begin{array}{l}\text { Amazonas } \\
\text { (Dept.) }\end{array}$ & $\mathrm{NI}(1 \mathrm{~N})$ \\
\hline & & & Apure & San Fernando (1F) \\
\hline & & & Aragua & Macay (1M); Anzortequi 1M) \\
\hline & & & Barinas & Calderas (13F, 7M); Macay (3M) \\
\hline & & & Carabobo & Independencia $(1 \mathrm{~F}, 1 \mathrm{M})$; \\
\hline & & & Cojedes & $\begin{array}{l}\text { Cohâra (1F); Limas Blancas (1F); } \\
\text { Manrrique (19F, 12M); San Carlos } \\
(7 \mathrm{M}) \text {; Tinaquillo (1F, 2M); NI (1F) }\end{array}$ \\
\hline & & & Falcón & PAHO insectary $(2 \mathrm{~F}, 1 \mathrm{M})$ \\
\hline & & & Lara & Barquisimeto (2M) \\
\hline & & Venezuela & Miranda & La Democracia $(1 \mathrm{~F}, 2 \mathrm{M})$ \\
\hline & & & Portuguesa & Papelón (5F, 3M); San Jorge (4F) \\
\hline & & & Trujillo & Pampán $(9 \mathrm{~F})$ \\
\hline & & & Yaracuy & San Felipe (3F, 4M) \\
\hline & & & NI & $\mathrm{NI}(1 \mathrm{M})$ \\
\hline & & Colombia & Putumayo & Puerto Assis (1M) \\
\hline & & & Portuguesa & Acarigua $(1 \mathrm{~F})$ \\
\hline & $p$ & & Tachira & San Simón (5F, 1M); Coloncito (3F) \\
\hline & tus robustus Larrousse, 1921 & Venezuela & Trujillo & Pampán (8F, 2M) \\
\hline & & & NI & NI $(15 \mathrm{~F}, 21 \mathrm{M})$ \\
\hline & & & Anzóategui & San Cristóbal (1M) \\
\hline & & & Barinas & Andrés Eloy Blanco (6F; 2M) \\
\hline
\end{tabular}

COLVEC: Collection of Chagas Disease Vectors; M: male; F: female; N: nymph; NI: no information; SE: sets of eggs; Dept.: Department; PAHO: Pan American Health Organization. 
TABLE 2 - Contribution of triatomine fauna from other American countries to the COLVEC collection (Tribe Triatomini Jeannel, 1919). Information in parentheses includes the specimen number and sex.

\begin{tabular}{|c|c|c|c|}
\hline Species & Country & $\begin{array}{c}\text { Province, } \\
\text { Department or } \\
\text { State } \\
\end{array}$ & County (sex) \\
\hline Dipetalogaster maxima (Uhler, 1894) & Mexico & NI & $\mathrm{NI}(1 \mathrm{~F}, 1 \mathrm{M})$ \\
\hline \multirow{7}{*}{ Eratyrus cuspidatus Stål, 1859} & Colombia & Santander & $\mathrm{NI}(1 \mathrm{~F}, 1 \mathrm{M})$ \\
\hline & \multirow{4}{*}{ Venezuela } & Cojedes & Manrrique $(1 \mathrm{~N})$ \\
\hline & & Portuguesa & Ospino (1M) \\
\hline & & Maracay & $\mathrm{NI}(2 \mathrm{~N})$ \\
\hline & & Trujillo & Laguaca $(1 \mathrm{~N})$ \\
\hline & \multirow{2}{*}{ NI } & $\mathrm{NI}$ & $\mathrm{NI}(1 \mathrm{~F}, 1 \mathrm{M})$ \\
\hline & & NI & $\mathrm{NI}(5 \mathrm{~N})$ \\
\hline \multirow{8}{*}{ Eratyrus mucronatus Stål, 1859} & Bolivia & $\mathrm{La} \mathrm{Paz}$ & La Paz (1F) \\
\hline & \multirow{7}{*}{ Venezuela } & Aragua & NI (1M) \\
\hline & & Cojedes & San Carlos (1M) \\
\hline & & Lara & NI (6F) \\
\hline & & Maracay & NI (2SE) \\
\hline & & Portuguesa & $\mathrm{NI}(1 \mathrm{~F}, 1 \mathrm{M})$ \\
\hline & & Trujillo & $\mathrm{NI}(1 \mathrm{~F})$ \\
\hline & & NI & $\mathrm{NI}(1 \mathrm{M})$ \\
\hline Meccus longipennis (Usinger, 1939) & Mexico & $\mathrm{NI}$ & NI (1M) \\
\hline Meccus phyllosomus (Burmeister, 1835) & Mexico & Nayarit & NI $(1 \mathrm{~F}, 1 \mathrm{M})$ \\
\hline \multirow{2}{*}{ Panstrongylus chinai (Del Ponte, 1929) } & Peru & Piura & Piura $(3 \mathrm{~F}, 2 \mathrm{M})$ \\
\hline & NI & NI & Grant from UNIFESP insectary $(1 \mathrm{~F}, 1 \mathrm{M})$ \\
\hline \multirow{7}{*}{ Panstrongylus geniculatus (Latreille, 1811) } & Colombia & Antioquia & Amalfi (1F) \\
\hline & \multirow{2}{*}{$\begin{array}{c}\text { Costa } \\
\text { Rica }\end{array}$} & Heredia & Puerto Viejo de Sarapiquí (1M) \\
\hline & & NI & $\mathrm{NI}(1 \mathrm{M})$ \\
\hline & \multirow{4}{*}{ Venezuela } & Aragua & El Limón (1F); NI (1F) \\
\hline & & Carabobo & NI (1M) \\
\hline & & Cojedes & Manrrique (1F) \\
\hline & & $\mathrm{NI}$ & NI (1M) \\
\hline \multirow{2}{*}{ Panstrongylus herreri Wygodzinsky, 1948} & Peru & Lima & $\operatorname{Lima}(1 \mathrm{~F}, 1 \mathrm{M})$ \\
\hline & Venezuela & Portuguesa & Araure (1F) \\
\hline \multirow{3}{*}{ Panstrongylus rufotuberculatus (Champion, 1899) } & Colombia & Antioquia & Amalfi (1M); \\
\hline & \multirow{2}{*}{ Venezuela } & Aragua & El Limón (1F, 2M); NI (2F, 2M) \\
\hline & & Trujillo & $\mathrm{NI}(1 \mathrm{M})$ \\
\hline \multirow{4}{*}{ Psammolestes arthuri (Pinto, 1926) } & \multirow{4}{*}{ Venezuela } & Apure & Mantecal (1F, 3M) \\
\hline & & Cojedes & $\begin{array}{l}\text { La Coromoto }(1 \mathrm{~F}) \text {; Manrrique }(7 \mathrm{~F}, 9 \mathrm{M}, 13 \mathrm{~N}) \text {; } \\
\text { San Carlos }(1 \mathrm{~F}, 1 \mathrm{M})\end{array}$ \\
\hline & & Portuguesa & Araure (1M); Guanare (1F, 20N, 1SE) \\
\hline & & NI & NI $(1 \mathrm{M})$ \\
\hline Psammolestes coreodes Bergroth, 1911 & Bolivia & Santa Cruz & Santa Cruz $(3 \mathrm{~F}, 3 \mathrm{M})$ \\
\hline \multirow{5}{*}{ Triatoma dimidiata Usinger, 1944} & \multirow{2}{*}{ Colombia } & Santander & NI (1F, 1M) \\
\hline & & Magdalena & Santa Marta (1F) \\
\hline & Guatemala & NI & $\mathrm{NI}(1 \mathrm{~F}, 1 \mathrm{M})$ \\
\hline & Mexico & NI & NI $(1 \mathrm{~F}, 1 \mathrm{M})$ \\
\hline & $\mathrm{NI}$ & $\mathrm{NI}$ & NI $(3 \mathrm{~F}, 4 \mathrm{M})$ \\
\hline
\end{tabular}


TABLE 2 - Continuation.

\begin{tabular}{|c|c|c|c|}
\hline Species & Country & $\begin{array}{c}\text { Province, } \\
\text { Department or } \\
\text { State }\end{array}$ & County (sex) \\
\hline \multirow{2}{*}{$\begin{array}{l}\text { Triatoma garciabesi } \\
\text { Carcavallo, Cichero, Martínez, Prosen \& Ronderos, } 1967\end{array}$} & Argentina & $\begin{array}{l}\text { Santiago Del } \\
\text { Estero }\end{array}$ & Santiago Del Estero (1F) \\
\hline & NI & NI & $\mathrm{NI}(1 \mathrm{~F}, 4 \mathrm{M})$ \\
\hline Triatoma guasayana Wygodzinsky \& Abalos, 1949 & $\mathrm{NI}$ & NI & $\mathrm{NI}(3 \mathrm{~F}, 8 \mathrm{M})$ \\
\hline Triatoma guazu Lent \& Wygodzinsky, 1979 & NI & NI & NI $(1 \mathrm{M})$ \\
\hline \multirow{3}{*}{ Triatoma infestans $\mathrm{n}$. ssp. } & \multirow{2}{*}{ Bolivia } & Cochabamba & Cochabamba (193F, 155M) \\
\hline & & Santa Cruz & Santa Cruz (3F, 10M) \\
\hline & NI & NI & $\mathrm{NI}(2 \mathrm{~F}, 3 \mathrm{M}, 1 \mathrm{~N})$ \\
\hline Triatoma lecticularia (Stål, 1859) & USA & Oklahoma & $\mathrm{NI}(1 \mathrm{~F}, 1 \mathrm{M})$ \\
\hline \multirow{5}{*}{ Triatoma maculata (Erichson, 1848) } & \multirow{5}{*}{ Venezuela } & Anzóategui & NI (2M) \\
\hline & & Cojedes & El Pao (1M); Manrique (2M) \\
\hline & & Lara & La Concepción (1F) \\
\hline & & Portuguesa & Araure (1F); Guanare (2F) \\
\hline & & Trujillo & Pampán (1F, 6M) \\
\hline \multirow{3}{*}{ Triatoma nigromaculata (Stål, 1872) } & \multirow{3}{*}{ Venezuela } & Aragua & El Limón (1M); NI (1F, 1M) \\
\hline & & Trujillo & NI $(1 M)$ \\
\hline & & Yaracuy & Nirgua $(3 \mathrm{M})$ \\
\hline Triatoma platensis Neiva, 1913 & NI & NI & NI $(1 \mathrm{M})$ \\
\hline Triatoma protracta (Uhler, 1894) & USA & California & $\mathrm{NI}(1 \mathrm{~F}, 1 \mathrm{M})$ \\
\hline \multirow{2}{*}{ Triatoma rubrovaria (Blanchard, 1843) } & Uruguay & NI & $\mathrm{NI}(2 \mathrm{~F}, 7 \mathrm{M})$ \\
\hline & NI & NI & NI $(1 \mathrm{M})$ \\
\hline \multirow{2}{*}{ Triatoma sordida (Stål, 1859) } & Bolivia & Santa Cruz & Santa Cruz (4F, 10M) \\
\hline & Venezuela & Portuguesa & Guanare $(\mathrm{N})$ \\
\hline
\end{tabular}

COLVEC: Collection of Chagas Disease Vectors; M: male; F: female; N: nymph. NI: No information; SE: sets of eggs; UNIFESP: Universidade Federal de São Paulo; USA: United States of America.

The list presents records of 4,778 specimens in CRIA's database. To date, there are 148 species of triatomine recognized ${ }^{9-15}$, of which 56 have representatives in the COLVEC collection and come from different geographic origins. Most of the collection comprises autochthonous species from Brazil, whose current triatomine fauna includes more than 60 species $^{11,16,17}$. Of these species, $35(57 \%)$ are registered in COLVEC, highlighting the taxonomic relevance of the collection.

The geographic distribution of Panstrongylus lutzi did not previously include the State of Minas Gerais; therefore, this list also helps extend the known occurrence of this vector, which affects the municipalities of Januária and Pirapora. Until recently, this species was mistaken for Panstrongylus geniculatus, which may occur more broadly because there are no reports of $P$. lutzi in the municipalities of Pirapora and Januária. Therefore, the need to establish greater interactivity between service laboratories and the reference laboratory must be emphasized. Moreover, two points are fundamental to lab staff members operating within the Chagas Disease Control Program: 1) participation in training courses on the identification of triatomines offered by LATEC in partnership with COLVEC and 2) assembling service collections with representatives of the local triatomine fauna.

Aimed at strengthening, complementing and expanding the collection, the objective of this study is to contribute to current and further studies and, primarily, ensure the ex situ conservation of triatomine fauna. 
TABLE 3 - List of Brazilian autochthonous Triatominae species (Hemiptera: Reduviidae) in the COLVEC collection. (Tribe Cavernicolini Usinger, 1944 and Tribe Rhodniini Pinto, 1926).

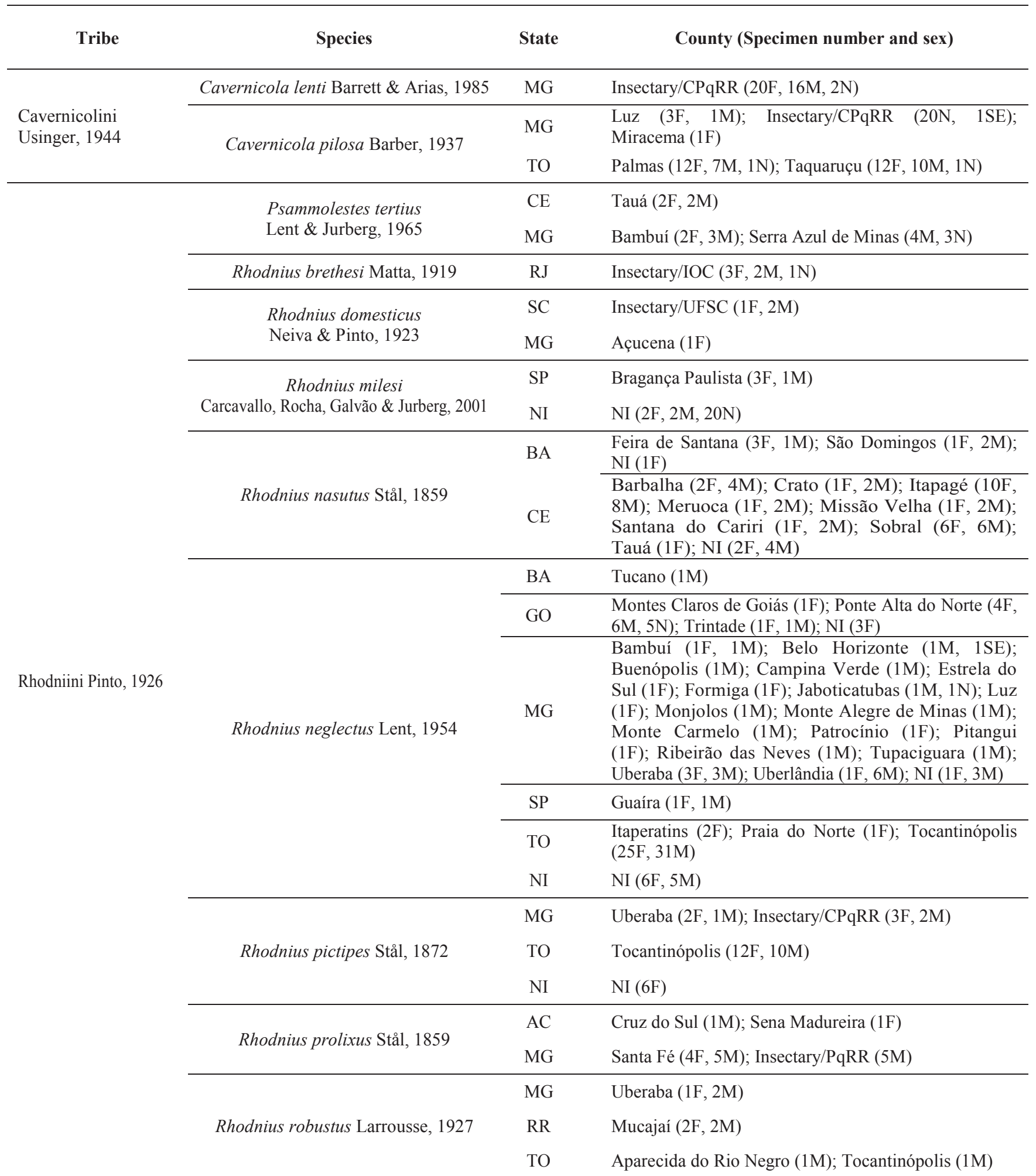

COLVEC: Collection of Chagas Disease Vectors; CPqRR: Centro de Pesquisa René Rachou; IOC: Instituto Oswaldo Cruz; UFSC: Universidade Federal de Santa Catarina; USP: Universidade de São Paulo; M: male; F: female; N: nymph; NI: no information; SE: set of eggs; MG: Minas Gerais; TO: Tocantins; CE: Ceará; RJ: Rio de Janeiro; SP: São Paulo; SC: Santa Catarina; BA: Bahia; GO: Goiás; AC: Acre; RR: Roraima. 
TABLE 4 - List of Brazilian autochthonous Triatominae species (Hemiptera: Reduviidae) in the COLVEC collection. (Tribe Triatomini Jeannel, 1919).

\begin{tabular}{|c|c|c|}
\hline Species & State & County (Specimen number and sex) \\
\hline \multirow[t]{3}{*}{ Panstrongylus diasi Pinto \& Lent, 1946} & MG & $\begin{array}{l}\text { Aimorés (2M); Berilo (1M); Braúnas (8N); Campos Gerais (1F); } \\
\text { Candeias (1F); Chapada do Norte (1M); Cláudio (1F, 2M); } \\
\text { Conceição do Pará (1M); Diamantina (1M); Divinópolis (1M); } \\
\text { Formiga (1F); Francisco Badaró (1F, 1M); Grupiara (1M); Itaguara } \\
\text { (1F); Itatiaiuçu (1F); Ituiutaba (1M); Luz (1M); Medeiros (1F); } \\
\text { Mirabela (1M); Pains (1F); Pará de Minas (1F); Pedra do Indaiá } \\
\text { (1F); Perdigão (2F); Pitangui (1F); Rio Pardo de Minas (1M); } \\
\text { Santo Antonio Amparo (1F); São João do Paraíso (1F, 3M); São } \\
\text { Sebastião do Oeste (2F); Veríssimo (1M); NI (1F, 4M) }\end{array}$ \\
\hline & $\mathrm{BA}$ & Pau Brasil (1F) \\
\hline & $\mathrm{CE}$ & Meruoca (3M) \\
\hline
\end{tabular}
(1F, 3M); Comercinho (1F, 1M); Conceição Mato Dentro (1M); Diamantina (1F, 2M); Felício dos Santos (2M); Francisco Badaró (4M); Itanhomi (2F, 1M); Ituiutaba (1M); Presidente Kubitschek (1M); Riacho dos Machados (1M); Rio Pardo de Minas (3M); Santa Fé (1M); São João do Paraíso (2F, 5M); Turmalina (1M); Uberlândia (1M); Unaí (1F, 1M); Veríssimo (1M); Virgem da Lapa $(1 \mathrm{M})$

\begin{tabular}{|c|c|c|}
\hline & & \\
\hline & TO & Lizarda (1M) \\
\hline & $\mathrm{NI}$ & $\mathrm{NI}(2 \mathrm{~F})$ \\
\hline Panstronovlus lionarius (Walker 1873) & RJ & Insectary/IOC $(17 \mathrm{~F}, 35 \mathrm{M})$ \\
\hline Panstrongylus lignarlus (walker, 18/3) & NI & $\mathrm{NI}(1 \mathrm{~F})$ \\
\hline
\end{tabular}

CE 35M); Independência $(6 \mathrm{~F} ; 1 \mathrm{M})$; Ipueiras $(1 \mathrm{M})$; Itapiúna $(1 \mathrm{M})$;

Massapê (3F); Meruoca (5F); Morada Nova (1M); Pacatuba (1M);

Panstrongylus lutzi (Neiva \& Pinto, 1923) Redenção (3M); Sobral (19F, 25M); Tauá (2F, 3M)

\begin{tabular}{cl}
\hline MG & Januária (1M); Pirapora (3F, 27M) \\
\hline PE & Orobó (1F, 2M) \\
\hline NI & NI (1F) \\
\hline AL & Palmeira dos Índios (1F, 1M) \\
\hline BA & $\begin{array}{l}\text { Campo Formoso (5F, 4M); Castro Alves (2F, 3M); Jequié (1F); } \\
\text { Salvador (1F); Santa Luzia (1F) }\end{array}$ \\
\hline CE & $\begin{array}{l}\text { Alcântaras (2F, 3M); Crateús (2F, 1M, 1N); Ipoeiras (1F); Meruoca } \\
(10 \mathrm{~F}, 10 \mathrm{M})\end{array}$ \\
\hline GO & Corumbá de Goiás (2F; 2M) \\
\hline & Araguari (1F); Bambuí (3F, 4M); Belo Horizonte (8F, 19M, 8N);
\end{tabular}
Araguari (1F); Bambuí (3F, 4M); Belo Horizonte (8F, 19M, 8N); Berilo (1F, 2M); Betim (2F, 1M); Brumadinho (72F, 93M); Carmo do Paranaíba (4F, 5M); Carmópolis (4F, 1M); Carmópolis de Minas (14F, 17M); Cascalho Rico (1M); Claro dos Poções (1F); Contagem (1M); Coromandel (1F, 1M); Douradoquara (1M); Estrela do Sul $(1 \mathrm{~F}, 2 \mathrm{M}, 1 \mathrm{~N})$; Guaxupé $(1 \mathrm{~F}, 1 \mathrm{M})$; Ibirité $(1 \mathrm{~F})$; Indianápolis $(1 \mathrm{M})$; Iraí de Minas (8F, 4M); Itanhomi (4F); Jaboticatubas (16F, 12M, $14 \mathrm{~N})$; Lagoa Grande (3N); Lagoa Santa (1M); Monjolos (1F, 3M); MG Monte Carmelo (1F, 3M, 1N); Monte Santo de Minas (1F, 1M); Morro da Garça (5F, 1M); Nova União (1F); Olhos-d'Água (1F, 1M); Patos de Minas (5F, 1M); Patrocínio (10F, 9M); Pedra do Indaiá (1F, 5N); Piracema (13F, 15M, 1N); Pitangui (7F, 10M, 17N); Ribeirão das Neves (2F); Rio Casca (1M); Rio Doce (1F); Rio Paranaíba (16F, 1M, 1N); Santa Cruz do Escalvado (5F, 5M); Santa Luzia (1M); Santa Maria do Suaçuí (3F); Santana do Riacho (4F, 5M); São Gotardo (3F); São José do Goiabal (1F); SSM (2M); SemPeixe (1F, 1M); Sete Lagoas (1F); Uberlândia (6F, 6M); Unaí (1N); Vazante (1F); Insectary/CPqRR (1F, 2M); NI (5F, 4M)

\begin{tabular}{ll}
\hline SC & Costeira do Pirajubaí $(1 \mathrm{~F}, 1 \mathrm{M}) ;$ Florianópolis $(1 \mathrm{~F}, 1 \mathrm{M}) ;$ NI $(7 \mathrm{~F}, 8 \mathrm{M})$ \\
\hline SP & Juquiá $(1 \mathrm{~F}, 1 \mathrm{M})$; Caconde $(1 \mathrm{~F}, 1 \mathrm{M})$ \\
\hline NI & NI $(1 \mathrm{M})$
\end{tabular}


TABLE 4 - Continuation.

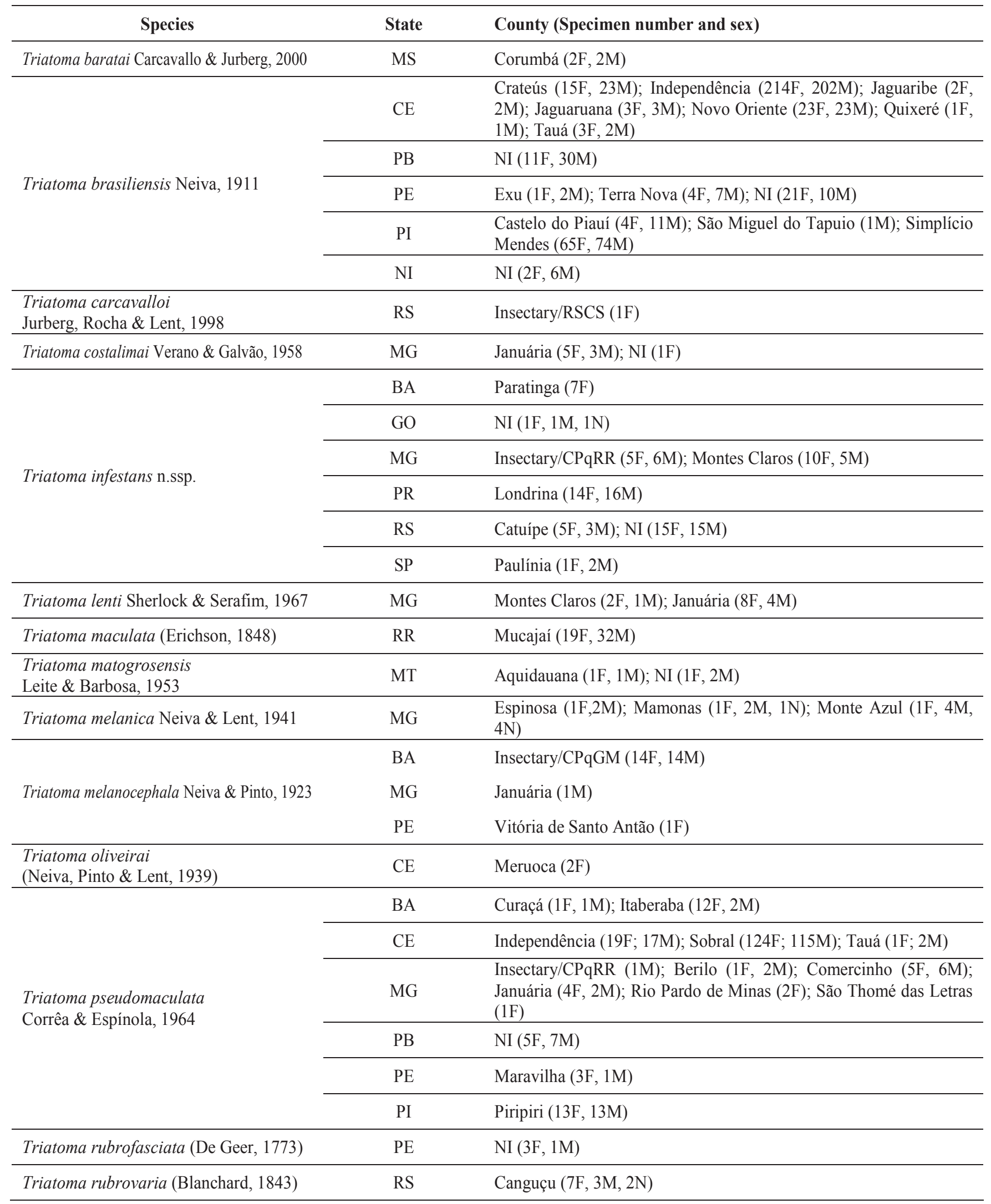


TABLE 4 - Continuation.

\begin{tabular}{|c|c|c|}
\hline Species & State & County (Specimen number and sex) \\
\hline \multirow{5}{*}{ Triatoma sordida (Stål, 1859) } & BA & Aracatu (4F, 3M); Livramento de Nossa Senhora (4F, 5M, 2N) \\
\hline & MS & Corumbá (13F; 7M) \\
\hline & MG & $\begin{array}{l}\text { Araguari (3F, 1M, 3N); Augusto de Lima (2F); Insectary/CPqRR } \\
\text { (6F, 6M); Buenópolis (25F, 5M, 2N); Buritis (3F, 2M, 1N); } \\
\text { Campina Verde (1F, 11N); Capinopólis (5F, 6M, 58N); Cônego } \\
\text { Marinho (1F, 1M, 2N); Coração de Jesus (3F, 2M, 1N); Curvelo } \\
\text { (4F, 3M, 4N); Douradoquara (7F, 2N, 1M); Espinosa (3F, 3M); } \\
\text { Estrela do Sul (2F); Grupiara (3F, 1M, 1N); Gurinhatã (7F, 8M); } \\
\text { Inimutaba (2M); Ipiaçu (1F, 1M); Januária (3F, 3M); Lagoa Grande } \\
\text { (26F, 21M, 22N); Limeiro do Oeste (3F, 3M); Luislândia (2F, 2M) } \\
\text { Manga (3F, 3M); Mato Verde (9F, 3M, 2N); Monjolos (8F, 1M, } \\
\text { 2N); Montalvânia (4F, 4M); Monte Alegre de Minas (1F); Montes } \\
\text { Claros (18F, 20M); Morro da Garça (1F); Porteirinha (3F, 3M); } \\
\text { Santo Hipólito (10F, 1M); São Francisco (5F, 6M); São João da } \\
\text { Lagoa (1N); Tupaciguara (1F, 1M); Uberaba (15F, 14M); } \\
\text { Uberlândia (1F, 3N); Uná (4F, 2M, 1N); Várzea da Palma (2F, 2M, } \\
\text { 2N); Varzelândia (3F, 3M); Verdelândia (23F, 13M); NI (12F, 8M, } \\
\text { 10N) }\end{array}$ \\
\hline & TO & Conceição do Tocantins (1F) \\
\hline & $\mathrm{NI}$ & $\mathrm{NI}(3 \mathrm{~F}, 1 \mathrm{M}, 1 \mathrm{~N})$ \\
\hline \multirow{2}{*}{ Triatoma tibiamaculata (Pinto, 1926) } & SP & IFSP/USP (1F, 1M) \\
\hline & NI & $\mathrm{NI}(1 \mathrm{~F}, 1 \mathrm{M})$ \\
\hline Triatoma vitticeps (Stål, 1859) & MG & $\begin{array}{l}\text { Açucena (3F, 1M, 2N, egg); Aimorés (1F, 1M); Almenara (1F, 1M, } \\
\text { 92N, 18SE); Araponga (3F); Insectary/CPqRR (4F, 5M); Caratinga } \\
\text { (1F); Comercinho (50F, 58M, 9N); Coronel Murta (1F); Diamantina } \\
\text { (7M); Divino (2F, 4M); Galiéia (1F); Itanhomi (92F, 64M); } \\
\text { Jaboticatubas (1F); Januária (2F, 2M); José Gonçalves de Minas } \\
\text { (1F, 2M); Juramento (2M); Mendes Pimentel (5N, 3SE); Riacho dos } \\
\text { Machados (1F, 2M); Rio Pardo de Minas (2F, 1M); Santa Cruz do } \\
\text { Escalvado (1M); Santana do Riacho (2F, 3M); SGRP (1M); São } \\
\text { João do Oriente (2F, 1M); São João do Pacuí (1M); Tabaúna (1F); } \\
\text { Teófilo Otoni (2F, 2M); Ubaí (1M); Virgolândia (11N) }\end{array}$ \\
\hline $\begin{array}{l}\text { Triatoma williami } \\
\text { Galvão, Souza \& Lima, } 1965\end{array}$ & MS & Corumbá (8F, 7M) \\
\hline Triatoma wygodzinskyi Lent 1951 & SP & São João da Boa Vista (1F, 2M) \\
\hline
\end{tabular}

COLVEC: Collection of Chagas Disease Vectors; M: male; F: Female; N: nymph; NI: No information; SE: set of eggs; CPqRR: Centro de Pesquisa René Rachou; IOC: Instituto Oswaldo Cruz; UFSC: Universidade Federal de Santa Catarina; CPqGM: Centro de Pesquisa Gonçalo Moniz; IFSP: Instituto Faculdade Saúde Pública; USP: Universidade de São Paulo; MG: Minas Gerais; CE: Ceará; TO: Tocantins; RJ: Rio de Janeiro; BA: Bahia; GO: Goiás; SP: São Paulo; AC: Acre; RR: Roraima; PE: Pernambuco; AL: Alagoas; SC: Santa Catarina; MS: Mato Grosso do Sul; PB: Paraíba; PI: Piauí; RS: Rio Grande do Sul; PR: Paraná; MT: Mato Grosso; RSCS: Regional de Santa Cruz do Sul; SSM: São Sebastião do Maranhão; SGRP: São Gonçalo do Rio Preto. 


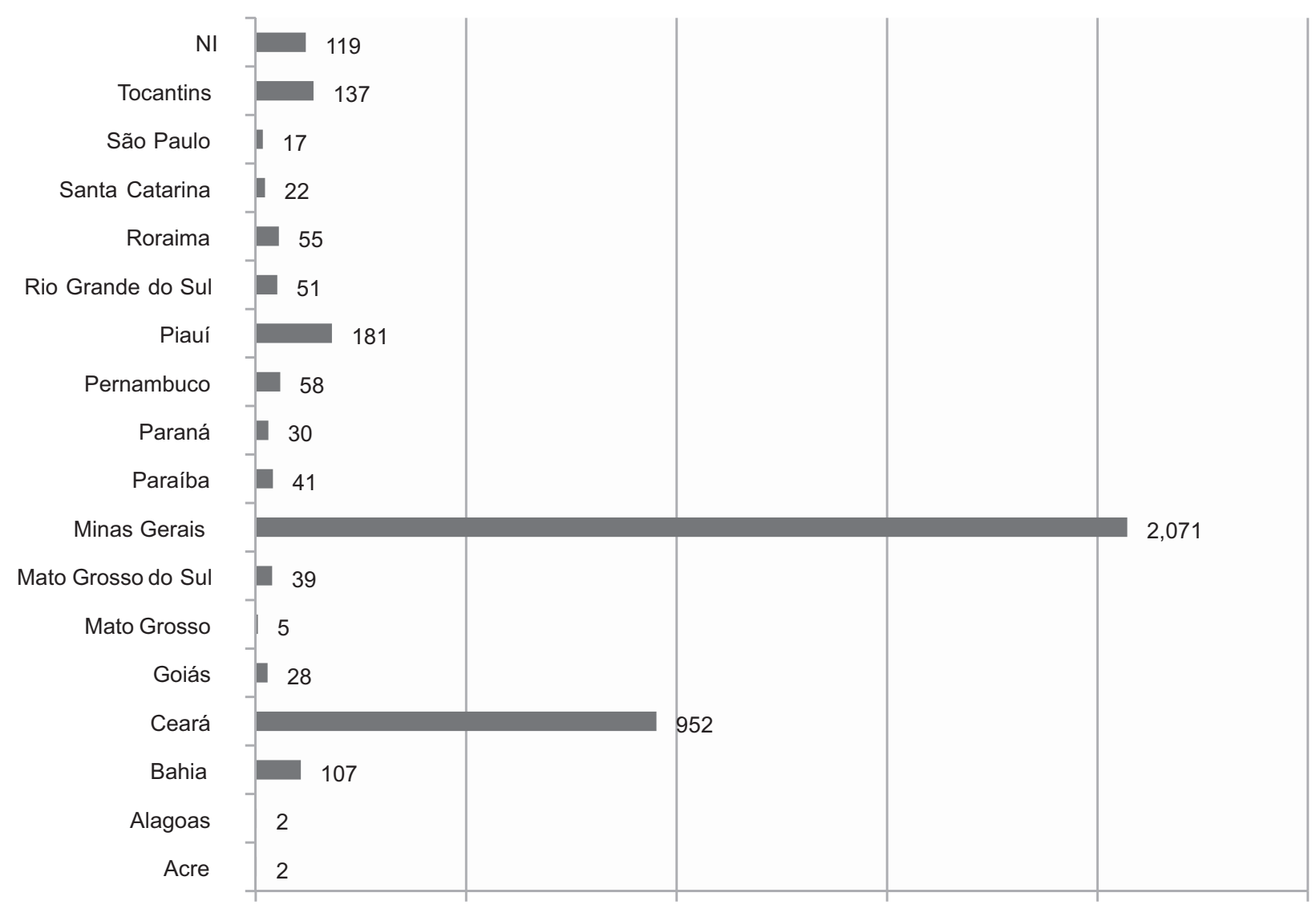

FIGURE 1 - Current number of COLVEC's entries per Brazilian State. NI: no information; COLVEC: Collection of Chagas Disease Vectors.

\section{CONFLICT OF INTEREST}

The authors declare that there is no conflict of interest.

\section{REFERENCES}

1. Silveira FA, Alvarenga AS. O acervo de abelhas da Coleção entomológica das coleções Taxonômicas da UFMG. MG BIOTA 2012; 4:5-24.

2. Silva M, Cupolillo E, Pirmez C. Gestão das Coleções Biológicas da Fundação Oswaldo Cruz. In: Anais do I Seminário sobre gestão e curadoria de Coleções Zoológicas da Fiocruz. Rio de Janeiro: Ed: Corbã; 2011. p. 25-26.

3. Marinoni L. Redes de informação nacionais e internacionais sobre Biodiversidade. In: Anais do I Seminário sobre gestão e curadoria de Coleções Zoológicas da Fiocruz. Rio de Janeiro: Ed: Corbã; Rio de Janeiro. 2011. p. 49.

4. Vale. Reserva Natural. Coleções. Belo Horizonte. [Cited 2010 April 17]. Available at: http://www.vale.com/reserva_natural_vale/ pesquisa_colecoes.asp/.

5. Romero Sá M. As coleções biológicas e o desenvolvimento das pesquisas biomédicas no Brasil. In: Anais do II Simpósio Nacional de coleções científicas. Rio de Janeiro: Ed: Corbã; 2008. p. 86-87.

6. Apfelbaum B. Guide to environmental protection of Colletions. Madison: Sound View Press; 1991.
7. Rose CL, Hawks CA, Genoways HH. Storage of natural history collections: a preventive conservative approach. In: Society for Preservation of Natural History Colletins. $2^{\text {rd }}$ ed. New York: York Graphics; 1995.

8. Malaro MC. A Legal Primer on Managing Museum Collections. Smithsonian Institution Press. Washington, DC: 1985. Available from American Association of Museums, Washington, DC. http:// www.aam-us.org.

9. Schofield CJ, Galvão C. Classification, evolution and species groups within the Triatominae. Acta Tropica 2009; 110:88-100.

10. Rosa JA, Rocha CS, Gardim S, Pinto MC, Mendonça VJ, Ferreira Filho JCR, et al. Description of Rhodnius montenegrensis n. sp. (Hemiptera: Reduviidae: Triatominae) from the State of Rondônia, Brazil. Zootaxa 2012; 3478:62-76.

11. Abad-Franch F, Pavan MG, Jaramillo NO, Palomeque FS, Dale C, Chaverra D, et al. Rhodnius barretti, a new species of Triatominae (Hemiptera: Reduviidae) from western Amazonia. Mem Inst Oswaldo Cruz 2013; 108 (supl I):92-99.

12. Costa J, Correia NC, Neiva VL, Gonçalves TC, Felix M. Revalidation and redescription of Triatoma brasiliensis macromelasoma Galvão, 1956 and an identification key for the Triatoma brasiliensis complex (Hemiptera: Reduviidae: Triatominae). Mem Inst Oswaldo Cruz 2013; 108:785-789.

13. Gonçalves TCM, Teves-Neves SC, Santos-Mallet JR, Carbajalde-la-Fuente AL, Lopes CM. Triatoma jatai sp. nov. in the state of Tocantins, Brazil (Hemiptera: Reduviidae: Triatominae). Mem Inst Oswaldo Cruz 2013; 108:429-437. 
14. Jurberg J, Cunha V, Cailleaux S, Raigorodschi R, Lima MS, Rocha DS, et al. Triatoma pintodiasi sp. nov. of the T. rubrovaria subcomplex (Hemiptera, Reduviidae, Triatominae). Rev PanAmazônica Saúde 2013; 43-56.

15. Poinar Jr G. Panstrongylus hispaniolae sp. n. (Hemiptera: Reduviidae: Triatominae), a new fossil triatomine in Dominican amber, with evidence of gut flagellates. Palaeodiversity 2013; $6: 1-8$.
16. Carcavallo RU, Girón IG, Jurberg J, Galvão C, Lent H. Chaves gráficas para as tribos, gêneros e espécies da subfamília Triatominae. In: Carcavallo RU, Girón IG, Jurberg J, Lent H, editors. Atlas dos Vetores da Doença de Chagas na Américas. Rio de Janeiro: FIOCRUZ, Press; vol 1, 1998 p. 107-244.

17. Gurgel-Gonçalves R, Galvão C, Costa J, Peterson AT. Geographic Distribution of Chagas Disease Vectors in Brazil Based on Ecological Niche Modeling. J Trop Med 2012; ID 705326. 